\title{
Fundamental Performance Trade-offs in Coexisting Wireless Networks
}

\author{
Keivan Navaie and Tuan Anh Le \\ School of Electronic and Electrical Engineering \\ University of Leeds, Leeds, UK, LS2 9JT \\ Email: \{keivan.navaie, tuan.a.le\}@ieee.org
}

\begin{abstract}
This paper considers coexisting wireless networks sharing radio resources with contradicting performance objectives. A multi-objective optimization framework is proposed to investigate the fundamental trade-off between the performance of the coexisting networks. For a given set of radio resources, the optimal achievable performances of the coexisting networks are characterize by a multi-dimensional Pareto set. If the performance objectives of some of coexisting networks are modified or adjusted, the proposed framework provides the maximum corresponding achievable variation to the performance of the rest of coexisting networks. The quantitative information provided by the proposed framework facilitates joint design and optimization of coexisting networks and can be further exploited for regulating the collaborations among coexisting networks. We further show that the corresponding Pareto set can be in fact engineered by adjusting the technologies adopted in each of these networks. We then apply the proposed framework to two coexisting cellular networks. An improvement of $25 \%$ on the achievable rate is observed in return of a slightly higher interference tolerance at one of the coexisting networks.
\end{abstract}

\section{INTRODUCTION}

Coexisting wireless networks scenario provides many opportunities for improving spectrum usage for the fifth generation (5G) wireless communication networks [1]. In the related literature however, there is no concise analytical tool for investigating the performance trade-offs in such a system. In this paper, we formulate the function of a system comprising coexisting networks as a multi-objective optimization problem (MOP) to investigate its fundamental performance trade-offs. The corresponding MOP includes a set of contradicting objectives such as maximizing achievable throughput, minimizing delay and minimizing outage probability. Since achieving such objectives in any of the coexisting wireless networks require allocating a larger portion of the shared radio resources, in principle it compromises the performance of the other coexisting networks.

Multi objective optimization theory introduces techniques that simultaneously optimize multiple yet contradicting objective functions [2], [3]. For MOP, instead of a single solution, there exists a set of optimal solutions characterize by Pareto optimality that simultaneously optimizes all contradicting objectives [3]. Here, we show that the corresponding Pareto set for coexisting wireless network provides essential quantitative information which can be used in design and management of collaboration between multiple coexisting operators sharing the same radio resources. We further provide examples to highlight how this framework can be used to establish a theoretical ground for collaboration between coexisting networks.

The Pareto set, also referred to as Pareto frontier, characterises the achievable performance of the multiple systems based on the available shared radio resources, the resource constraints in each of the coexisting networks, and their corresponding quality-of-service requirements. We further show that the Pareto frontier can be engineered by adjusting the technologies adopted in each of these networks. This can facilitate joint design of the multiple networks to maximize the utilization of the shared radio resources.

Multi objective optimization is utilized in different engineering applications, see, e.g., [4] and references therein. MOP is adopted [5] as a tool for designing routing algorithms in ad-hoc networks. An applications of MOP in radio resource scheduling in cellular networks is also presented in [6] where, MOP is used to develop a distributed resource allocation scheme. Further in [7] MOP is utilized in beamforming design for secure communications in a wireless system. In [8] MOP approach is used for formulation of the optimal link adaptation problem of orthogonal frequency division multiplexing in a cognitive radio system, where secondary users can opportunistically access the spectrum of primary users. MOP model in [8] is developed based on joint maximizing the cognitive system throughput and minimizing its transmit power. As it is seen, in the related literature MOP has been used as an algorithmic tool which facilitates developing new algorithms for single networks. To the best of our knowledge, this paper for the first considers MOP as a modeling too for coexisting networks to investigate their fundamental performance trade-offs.

To show the application of the proposed framework here we also present cognitive beamforming as a case study in which two systems, primary and cognitive (also referred to as secondary) share the same spectrum. The secondary system is facilitated by multiple antenna and capable of beamforming. We then formulate the function of this spectrum sharing system using MOP with a set of contradicting objectives. One objective is to minimize the interference due cognitive base station (BS) transmission on the primary system receivers. Maximizing the intended signal received at all cognitive users 
are also considered as performance objectives in this system.

Using the analytical tools provided by the multi objective optimization theory, we then derive an equivalent standard semi-definite programming form with adjustable parameters that facilitate system design. The obtained solution set provides the best achievable throughput (characterized by the maximum intended signal power at each cognitive user) for a given level of interference tolerance in the primary system. It also evaluates the maximum achievable gain on the system throughput if the primary system is able to make compromises and increase its interference tolerance, such framework could be a base for negotiations between the operators. Note that in practice reaching to the optimal points might be hard even impossible. Nevertheless it provides the best each network can achieve out of the available resources in a given setting. Simulation results show that a possible improvement of $10 \mathrm{bits} / \mathrm{s} / \mathrm{channel}-\mathrm{use}$ in the secondary system total throughput is achievable in return of a slightly higher interference threshold in the primary system.

The organization of the paper is as the following. In Section II, the preliminary definitions for multi objective optimization as well as the system model are presented. Based on a practical example we further discuss engineering of Pareto frontier in Section III . Then in Section IV we present a case study followed by simulation results. The paper is concluded in Section V.

Notations: $\operatorname{Tr}(\cdot)$ : trace operator; $\mathbf{Y} \succeq 0$ : a positive semi definite matrix; $\preccurlyeq$ : element-wise inequality; $\left(y_{i}\right)_{i=1}^{U}$ : $\left[\begin{array}{llll}y_{1} & y_{2} & \cdots & y_{U} ;\end{array}\right]^{T} ; \mathbb{E}(x)$ : expected value of random variable $x$.

\section{SyStem Model AND PRELIMINARIES}

We consider a system consisting of $P$ objective functions indexed by $p, f_{p}(\mathbf{x})$, where $\mathbf{x}$ is the resource allocation vector/matrix. Examples of the objective function $f_{p}(\mathbf{x})$ include maximum delay, outage probability, or a combination of different key performance indicators in the coexisting networks such as a utility function.

The function of this system is modelled as a multi objective optimization problem (MOP) as the following:

$$
\min _{\mathbf{x} \in \mathcal{X}} \mathbf{f}(\mathbf{x})=\min _{\mathbf{x} \in \mathcal{X}}\left(f_{1}(\mathbf{x}), \cdots, f_{p}(\mathbf{x})\right),
$$

where $\mathcal{X}$ and $\mathbf{f}(\mathbf{x}) \in \mathbb{R}^{p}$ are the resource allocation space, and the objective space, respectively. In fact $\mathcal{X}$ characterise the solution space based on the available resources in the networks, the quality-of-service requirement for the network users, as well as the adopted air interface technology in each of the coexisting network.

For instance for a network with energy constraint providing bit-error-rate sensitive services, adopting an air interface technology with higher tolerance to interference (e.g, an air interface including sophisticated channel coding technique) results in a larger solution set comparing to the case where an interface technology with lower interference tolerance has been adopted.

An MOP is further characterized by its feasible set, objective function vector $\mathbf{f}$, and the objective space. The objective function vectors $\mathbf{y}=\mathbf{f}(\mathbf{x})$, i.e., $\mathbf{x} \in \mathcal{X}$, are mapped from $\mathbb{R}^{p}$ to an ordered space, e.g. $\left(\mathbb{R}^{p}, \preccurlyeq\right)$, where comparisons are made using the order relation. This mapping is called the model map, $\theta$. An MOP is completely described by

$$
\left(\mathcal{X}, \mathbf{f}, \mathbb{R}^{p}\right) / \theta /\left(\mathbb{R}^{p}, \preccurlyeq\right),
$$

which includes feasible set, objective function vector, objective space, model map and order space. An MOP class is the set of all MOPs with the same model map and order space which is denoted by $\bullet / \theta /\left(\mathbb{R}^{p}, \preccurlyeq\right)$.

\section{A. Solution Set and Optimality}

Here we consider Pareto optimality for a class of MOPs: -/i.d./ $\left(\mathbb{R}^{p}, \preccurlyeq\right)$, where the model map is an identical mapping (i.d.), i.e., $\theta(\mathbf{y})=\mathbf{y}$.

Consider the following MOP of the class $\bullet /$ i.d./ $\left(\mathbb{R}^{p}, \preccurlyeq\right)$ :

$$
\min _{\mathbf{x} \in \mathcal{X}}\left(f_{1}(\mathbf{x}), \cdots, f_{p}(\mathbf{x})\right) .
$$

Let $\mathcal{Y}=\mathbf{f}(\mathcal{X})$ be the image of the feasible set $\mathcal{X}$ under the objective function mapping $\mathbf{f}($.$) .$

Definition Pareto Optimality: A feasible solution $\widehat{\mathbf{x}} \in \mathcal{X}$ is called Pareto optimal if there is no other $\mathbf{x} \in \mathcal{X}$ such that $\mathbf{f}(\mathbf{x}) \preccurlyeq \mathbf{f}(\widehat{\mathbf{x}})$. If $\widehat{\mathbf{x}}$ is Pareto optimal then $\mathbf{f}(\widehat{\mathbf{x}})$ is said to be a non-dominated point. If $\mathbf{x}^{1}, \mathbf{x}^{2} \in \mathcal{X}$ and $\mathbf{f}\left(\mathbf{x}^{1}\right) \preccurlyeq \mathbf{f}\left(\mathbf{x}^{2}\right)$, then it is said that $\mathbf{x}^{1}$ dominates $\mathbf{x}^{2}$ and $\mathbf{f}\left(\mathbf{x}^{1}\right)$ dominates $\mathbf{f}\left(\mathbf{x}^{2}\right)$. The set of all Pareto optimal solutions, i.e., Pareto optimal set, $\widehat{\mathbf{x}} \in \mathcal{X}$ is represented by $\mathcal{X}_{E}$. Non-dominated set, is denoted by $\mathcal{Y}_{N}$, where all non-dominated points $\widehat{\mathbf{y}}=\mathbf{f}(\widehat{\mathbf{x}})$ are in $\mathcal{Y}$.

Pareto optimal set is also referred to as Pareto Frontier. Pareto optimality allows no improvement in any objective function is the set without trade-offs from the others.

\section{Performance Trade-OfFs: Pareto Frontier}

To have a better understanding, here we consider a system including two coexisting networks. Two contradicting objectives are: maximizing the throughput in the first network $\left(f_{1}(\mathbf{x})=-1 \times R\right)$, and minimizing the outage probability in the second network $\left(f_{2}(\mathbf{x})=P_{\text {Outage }}\right)$. Outage occurs if the received interference due to the second network activity is higher than a given interference threshold, $I_{t h}$. In such a system the Pareto frontier forms a curve as illustrated in Fig. 1.

As it is seen for a given outage probability $O_{1}$, the maximum theoretical achievable throughput for the second system is $R_{1}$. This provides a benchmark for comparison of the actual performance with that of the maximum achievable. Fig. 1 also indicates that the outage probability in the first system can be traded in return of an increase in the throughput of the second system: assume that first system is able to be more flexible on the outage probability, $\mathrm{O}_{2}$, the second system is then able to achieve the maximum rate of $R_{2}$, where $R_{2}>R_{1}$. An instance 


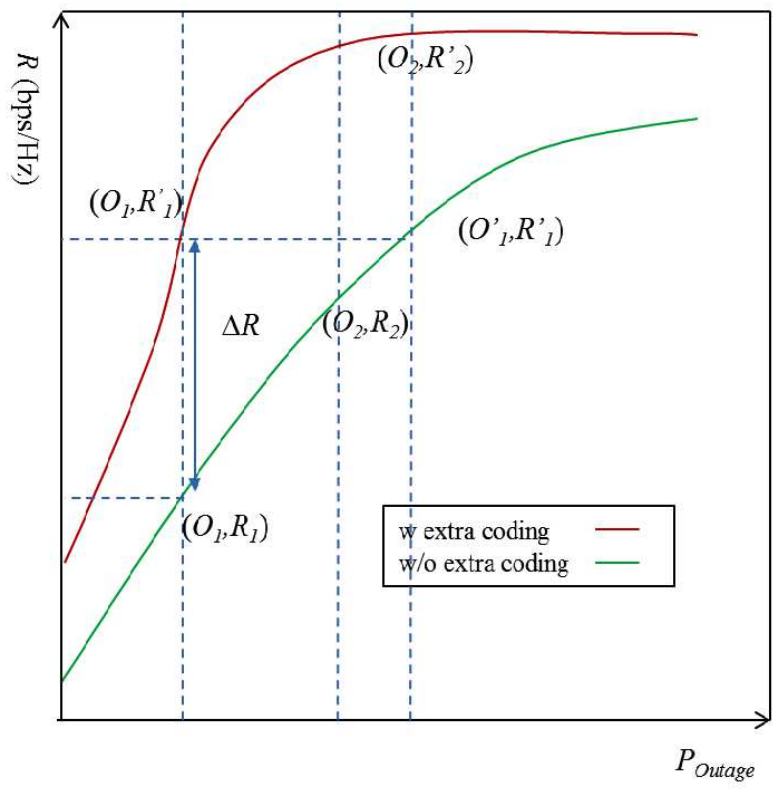

Fig. 1. An illustration of the Pareto frontier for two coexisting system with $f_{1}(\mathbf{x})=-1 \times R$, and $f_{2}(\mathbf{x})=P_{\text {outage }}$ in two cases of with and without extra channel coding.

of increasing outage tolerance is where a the first network has an interference limited air interface (e.g., 3G CDMA-based air interface) and shares the same spectrum with the second network. If the first network experiences a lower traffic load, thus for a given receiver performance, it is theoretically able to tolerate a higher level of interference from the second network, i.e., $I_{t h}^{\prime}$, and $I_{t h}^{\prime}>I_{t h}$. Tolerating a larger $I_{t h}^{\prime}$ can be translated to a higher outage probability with $I_{t h}$.

In the above example, a higher level of interference from the coexisting network can be also tolerated in the first network if it has an improved outage probability requirements. For instance, in [9] we design a sophisticated coding technique to improve the robustness of the first system to the received interference. Adopting such a coding scheme in the first network enables the second system to increase its throughput. Using new coding scheme in the first network accordingly modifies the Parto frontier of the system as shown in Fig. 1. In this case for given outage probability $O_{1}$ a higher throughput of $R_{1}^{\prime}$ is achieved in the second network. The Pareto frontier in Fig. 1 further provides the maximum achievable gain on the throughput in the second network, $\Delta R$. Based on Fig. 1 we can also evaluate the lowest possible outage probability in the first network, for a given throughput in the second network.

Modification of the Pareto frontier using channel coding in the above example is an instance of engineering the Pareto frontier by using new combination of physical layer technologies. Such modifications based on the tools provided by MOP model can help us to manage the compromises and achieve a higher spectral efficiency.

\section{Case Study: Cognitive Beamforming Design}

In this section we apply the above MOP framework to model the performance of a spectrum sharing system. In this system primary and secondary systems are coexisted cellular systems both access a shared spectrum. The secondary system is also cellular and utilizes underlay spectrum access [10]. In the secondary network, a cognitive base station (BS) supports $U$ secondary users sharing the uplink spectrum of the primary network, subject to the received interference threshold at the primary receivers.

The cognitive BS is equipped with $M$ antenna elements and capable of beamforming. For simplicity, we also assume single antenna setting at the secondary users (SUs) and the primary BS. The received signal at the $\mathrm{SU} i, i \in\{1, \cdots, U\}$, is

$$
y_{i}=\mathbf{h}_{s, i}^{H} \mathbf{w}_{i} s_{i}+\sum_{j=1, j \neq i}^{U} \mathbf{h}_{s, i}^{H} \mathbf{w}_{j} s_{j}+n_{i},
$$

where $\mathbf{h}_{s, i}^{H} \in \mathbb{C}^{1 \times M}$ is the channel between the cognitive BS and SU $i, \mathbf{w}_{i} \in \mathbb{C}^{M \times 1}$ is the beamforming vector for the SU $i, s_{i}$ is the data symbol to be sent to the $\mathrm{SU} i$ and $n_{i}$ is a zero mean circularly symmetric complex Gaussian noise with variance $\sigma_{i}^{2}$, i.e., $n_{i} \sim \mathcal{C N}\left(0, \sigma_{i}^{2}\right)$.

The primary network imposed interference at the SUs is considered as an additive background noise. For brevity the average transmitted symbol energy to $\mathrm{SU} i$ at the cognitive BS is assumed to be unity. Let $\mathbf{R}_{s, i}=\mathbb{E}\left(\mathbf{h}_{s, i} \mathbf{h}_{s, i}^{H}\right)$ and $\mathcal{W}=\left\{\mathbf{w}_{1}, \mathbf{w}_{2}, \cdots, \mathbf{w}_{U}\right\}$ be the set of candidate beamforming vectors in the cognitive BS for all SUs. The SINR at SU $i$ is

$$
g_{i}(\mathcal{W})=\frac{\mathbf{w}_{i}^{H} \mathbf{R}_{s, i} \mathbf{w}_{i}}{\sum_{j=1, j \neq i}^{U} \mathbf{w}_{j}^{H} \mathbf{R}_{s, i} \mathbf{w}_{j}+\sigma_{i}^{2}} .
$$

Let $\mathbf{R}_{p}=\mathbb{E}\left(\mathbf{h}_{p} \mathbf{h}_{p}^{H}\right)$, where $\mathbf{h}_{p}^{H} \in \mathbb{C}^{1 \times M}$ is the channel between the cognitive BS and the primary BS. Total interference at the primary BS induced by cognitive BS is $\sum_{i=1}^{U} \mathbf{w}_{i}^{H} \mathbf{R}_{p} \mathbf{w}_{i}$.

\section{A. MOP Formulation}

Here the objective is to maximize the intended signal power received at each $\mathrm{SU} i$ defined as

$$
f_{i}(\mathcal{W})=-\mathbf{w}_{i}^{H} \mathbf{R}_{s, i} \mathbf{w}_{i}
$$

while minimizing the interference imposed at the primary BS,

$$
f_{0}(\mathcal{W})=\sum_{i=1}^{U} \mathbf{w}_{i}^{H} \mathbf{R}_{p} \mathbf{w}_{i}
$$

The intended signal power received is directly lined to the achievable throughput. Therefore, the objective vector is

$$
\mathbf{f}(\mathcal{W})=\left(f_{0}(\mathcal{W}), f_{1}(\mathcal{W}), \cdots, f_{U}(\mathcal{W})\right)
$$


As it is seen the above formulation is in fact an MOP. Now, we define the decision space

$$
\begin{array}{r}
\mathcal{D} \triangleq\left\{\mathcal{W} \mid\left(\gamma_{i}\right)_{i=1}^{U} \preccurlyeq\left(g_{i}(\mathcal{W})\right)_{i=1}^{U}, \sum_{i=1}^{U} \mathbf{w}_{i}^{H} \mathbf{w}_{i} \leq P_{\mathrm{m}},\right. \\
\left.\sum_{i=1}^{U} \mathbf{w}_{i}^{H} \mathbf{R}_{p} \mathbf{w}_{i} \leq I_{\mathrm{m}}\right\},
\end{array}
$$

where $\gamma_{i}$ is the required SINR level at SU $i, P_{\mathrm{m}}$ is the cognitive BS maximum transmit power, and $I_{\mathrm{m}}$ is the interference threshold at the primary BS. We propose the following MOP class $\left(\mathcal{D}, \mathbf{f}, \mathbb{R}^{U+1}\right) /$ i.d./ $\left(\mathbb{R}^{U+1}, \preccurlyeq\right)$ :

$$
\min _{\mathcal{W} \in \mathcal{D}}\left(f_{0}(\mathcal{W}), f_{1}(\mathcal{W}), \cdots, f_{U}(\mathcal{W})\right)
$$

The intended signal received power at the secondary receiver is an indication of the maximum achievable rate. In practice for a given interference threshold at the primary receiver the solution set for (6) provides the maximum intended received signal power, i.e., the maximum achievable rate.

Using the tools provided by multi objective optimization theory, we then find the solutions to (6). We adopt weighted sum method [3] to obtain the solutions. Using this method (6) is reduced to a single objective problem as the following non-xonvex optimization:

\section{Problem $\mathcal{O} 1:$}

$$
\begin{array}{ll}
\min _{\mathcal{W}} & \lambda_{0} \sum_{i=1}^{U} \mathbf{w}_{i}^{H} \mathbf{R}_{p} \mathbf{w}_{i}-\sum_{i \in \mathcal{S}_{s}} \lambda_{i} \mathbf{w}_{i}^{H} \mathbf{R}_{s, i} \mathbf{w}_{i}, \\
\text { s. t. } & \frac{\mathbf{w}_{i}^{H} \mathbf{R}_{s, i} \mathbf{w}_{i}}{\sum_{j=1, j \neq i}^{U} \mathbf{w}_{j}^{H} \mathbf{R}_{s, i} \mathbf{w}_{j}+\sigma_{i}^{2}} \geq \gamma_{i}, \quad \forall i \\
& \sum_{i=1}^{U} \mathbf{w}_{i}^{H} \mathbf{w}_{i} \leq P_{\mathrm{m}}, \\
& \sum_{i=1}^{U} \mathbf{w}_{i}^{H} \mathbf{R}_{p} \mathbf{w}_{i} \leq I_{\mathrm{m}} .
\end{array}
$$

Here, we define beamforming matrix $\mathbf{W}_{i}=\mathbf{w}_{i} \mathbf{w}_{i}^{H}$, where $\mathbf{W}_{i} \succeq 0$ and $\mathbf{W}_{i}$ is a rank-one matrix. A matrix is rank one if its largest number of linearly independent columns/rows is one. Then, by rearranging the constraints, using $\mathbf{x}^{H} \mathbf{Y} \mathbf{x}=$ $\operatorname{Tr}\left(\mathbf{Y} \mathbf{x x}^{H}\right)$, and dropping the rank-one condition on $\mathbf{W}_{i},(7)$ is converted to:

\section{Problem 0 2:}

$$
\begin{array}{ll}
\min _{\left\{\mathbf{W}_{i}\right\}} & \lambda_{0} \operatorname{Tr}\left(\mathbf{R}_{p} \sum_{i=1}^{U} \mathbf{W}_{i}\right)-\operatorname{Tr}\left(\sum_{i=1}^{U} \lambda_{i} \mathbf{R}_{s, i} \mathbf{W}_{i}\right), \\
\text { s. t. } & k_{i}\left(\left\{\mathbf{W}_{i}\right\}\right) \geq 0, \mathbf{W}_{i} \succeq 0, \forall i \\
& P_{\mathrm{m}}-\sum_{i=1}^{U} \operatorname{Tr}\left(\mathbf{W}_{i}\right) \geq 0, \\
& \sum_{i=1}^{U} \mathbf{w}_{i}^{H} \mathbf{R}_{p} \mathbf{W}_{i} \leq I_{\mathrm{m}}, \\
& \mathbf{W}_{i} \succeq 0, \forall i
\end{array}
$$

where $\left\{\mathbf{W}_{i}\right\}=\left\{\mathbf{W}_{1}, \cdots, \mathbf{W}_{U}\right\}$ is the set of beamforming matrices and

$$
k_{i}\left(\left\{\mathbf{W}_{i}\right\}\right)=\operatorname{Tr}\left(\mathbf{R}_{s, i} \mathbf{W}_{i}\right)-\gamma_{i} \sum_{j=1, j \neq i}^{U} \operatorname{Tr}\left(\mathbf{R}_{s, i} \mathbf{W}_{j}\right)-\gamma_{i} \sigma_{i}^{2} .
$$

We then prove that $\mathcal{O} 1$ and $\mathcal{O} 2$ are equivalent. The proof is not provided here due to space limitation.

The optimization problem in $\mathcal{O} 2$ is a convex semi-definite programming [12], thus can be solved by the SeDuMi solver, provided by CVX optimization package [12], to obtain the set of optimal beamforming matrices $\mathbf{W}_{i}^{\star}$. Finally, the corresponding optimal solution to (7) is $\mathbf{w}_{i}^{\star}=\sqrt{\epsilon_{i}^{\star}} \mathbf{v}_{i}^{\star}$, where $\epsilon_{i}^{\star}$ and $\mathbf{v}_{i}^{\star}$ are the eigenvalue and the corresponding eigenvector of the rank-one matrix $\mathbf{W}_{i}^{\star}$, respectively [13].

\section{B. Simulation Results}

We simulate the scenario with a cognitive BS serving 2 SUs, i.e., $U=2$. The SUs are located at $-30^{\circ}$ and $50^{\circ}$ while the primary $\mathrm{BS}$ is located at $10^{\circ}$ relative to the array broadside of the cognitive BS. The distances from the SUs and primary BS to the cognitive BS are $0.5 \mathrm{~km}$ and $1 \mathrm{~km}$, respectively. The channel covariance matrices $\mathbf{R}_{s, i}$ and $\mathbf{R}_{p}$ are obtained using

$$
\mathbf{R}_{s, i}=\beta_{s, i} \mathbf{R}\left(\theta_{s, i}, \sigma_{a}\right) \text { and } \mathbf{R}_{p}=\beta_{p} \mathbf{R}\left(\theta_{p}, \sigma_{a}\right),
$$

respectively, where $\beta_{s, i}$ or $\beta_{p}$ represents the channel gain coefficient, $\theta_{s, i}$ or $\theta_{p}$ is the angle of departure, $\sigma_{a}$ is the standard deviation of the angular spread, and the $(p, q)$ th entry of $\mathbf{R}\left(\theta, \sigma_{a}\right)$ is, [13]:

$$
e^{\frac{j 2 \pi \Delta}{\lambda}[(q-p) \sin \theta]} e^{-2\left[\frac{\pi \Delta \sigma_{a}}{\lambda}\{(q-p) \cos \theta\}\right]^{2}} .
$$

In (9) $\beta_{s, i}$ or $\beta_{p}$ captures the distance-dependent path-loss according to $34.5+35 \log _{10}(l)$, where $l$ is the distance in meters with $l \geq 35 \mathrm{~m}$, a log-normal shadow fading with $8 \mathrm{~dB}$ standard deviation and a Rayleigh component for the multi-path fading channel. In (10), $\sigma_{a}=2^{\circ}$ and the antenna spacing at the BS $\Delta=\lambda / 2$, where $\lambda$ is the carrier wavelength. The subcarrier bandwidth, the noise power spectral density, the noise figure at each user receiver and antenna gain are assumed to be $15 \mathrm{kHz}$, $-174 \mathrm{dBm} / \mathrm{Hz}, 5 \mathrm{~dB}$ and $15 \mathrm{dBi}$, respectively. 


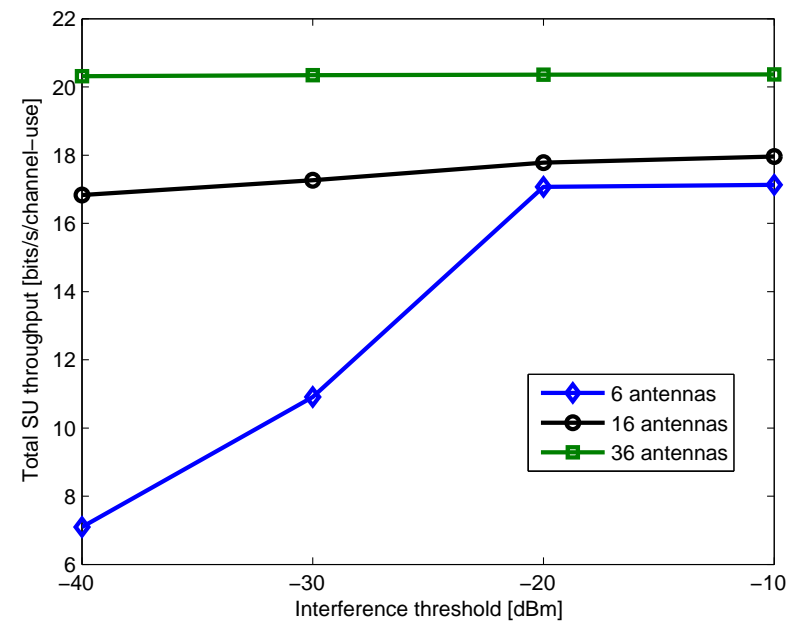

Fig. 2. Total SU throughput with different numbers of antenna elements at secondary BS vs. various interference thresholds, i.e., $I_{\mathrm{m}}$, at the primary BS. In (8), $P_{\mathrm{m}}=40 \mathrm{dBm}, \lambda_{0}=\lambda_{1}=\lambda_{2}=1 / 3$, and $\gamma_{1}=\gamma_{2}=10 \mathrm{~dB}$.

Fig. 2 illustrates an approximation of the Pareto frontier, where the total SU throughput with different numbers of antenna elements at the cognitive BS, versus various interference thresholds, i.e., $I_{\mathrm{m}}$, at the primary BS. In other words, for each given $I_{m}$, Fig. 2 gives the maximum achievable system throughput. It is also seen that in this case one way to engineer the Pareto frontier is modifying the number of antennas. For larger number of antennas, the total SU throughput becomes constant irrespective of interference threshold level at the primary BS. This is because the beamformer has a better resolution at higher number of antenna elements, i.e., see Fig. 3. As it is seen in Fig. 2 at the interference threshold of $-30 \mathrm{dBm}$ by engineering the Pareto frontier through increasing antenna elements to 16 , an improvement of almost $7 \mathrm{bits} / \mathrm{s} / \mathrm{channel-use}$ is achieved in the system throughput. In case of 6 antenna elements it is also seen that by increasing the interference threshold from $-23 \mathrm{dBm}$ to $-20 \mathrm{dBm}$ results in $25 \%$ improvement in the system achievable rate.

\section{CONCLUSION}

We proposed an analytical framework based on multi objective optimization for coexisting wireless networks accessing the same shared radio resources with contradicting performance objectives. The proposed framework shed light on the fundamental performance trade-offs in such system. We showed that for a given set of radio resources, the optimal achievable performances of the coexisting networks form a multi-dimensional Pareto set. If the performance objectives of some of coexisting networks are modified or adjusted, the proposed framework provides the maximum corresponding achievable variation to the performance of the other coexisting networks. We further showed that the corresponding Pareto front can be engineered by adjusting the technologies adopted

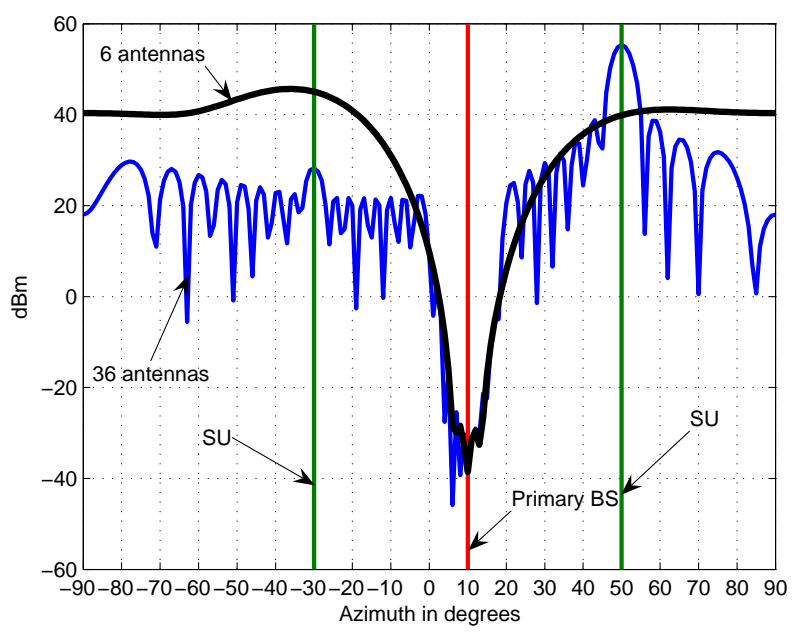

Fig. 3. Radiation pattern at the cognitive BS with 6 and 16 antenna elements. In (8), $I_{\mathrm{m}}=-40 \mathrm{dBm}, P_{\mathrm{m}}=40 \mathrm{dBm}, \lambda_{0}=\lambda_{1}=\lambda_{2}=1 / 3$, and $\gamma_{1}=\gamma_{2}=10 \mathrm{~dB}$.

in each of these networks to fit the design consideration in a coexisting environment. The proposed framework in this paper provides tools which facilitate joint design and optimization of coexisting networks to maximize the utilization of the shared radio resources. We the applied the proposed framework to the case of two coexisting cellular systems. It was shown through simulation that at the interference threshold of $-30 \mathrm{dBm}$ by engineering the Pareto frontier through increasing antenna elements to 16 , an improvement larger than $7 \mathrm{bits} / \mathrm{s} / \mathrm{channel-}$ use is achieved in the system throughput.

\section{ACKNOWLEDGMENT}

This work was partly supported by the UK Engineering and Physical Sciences Research Council (EPSRC) under grant EP/K/022725/1, and EU Marie Curie Career Integration Grant (MC-CIG) under grant 304086-S3isE.

\section{REFERENCES}

[1] L. Qian, H. Niu, A. Papathanassiou, and G. Wu, "5G network capacity: key lements and technologies," IEEE Vehicular Technology Magazine, vol. 9, no. 1, pp. 71-78, 2014.

[2] R. Devarajan, S. C. Jha, U. Phuyal, and V. K. Bhargava, "Energyaware resource allocation for cooperative cellular network using multiobjective optimization approach," IEEE Transactions on Wireless Communications, vol. 11, no. 5, pp. 1797-1807, May 2012.

[3] M. Ehrgott, Multicriteria Optimization. Springer, 2005.

[4] A. Johan. "Survey of multiobjective optimization in engineering design," Department of Mechanical Engineering, Linktjping University, Sweden, 2000.

[5] K. Jaffres-Runser, C. Comaniciu, and J. Gorce, "A multiobjective optimization framework for routing in wireless ad hoc networks," arXiv preprint arXiv:0902.0782, 2009.

[6] M. Elmusrati, H. El-Sallabi, and H. Koivo, "Applications of multiobjective optimization techniques in radio resource scheduling of cellular communication systems," IEEE Transactions on Wireless Communications, vol. 7, no. 1, pp. 343-353, 2008 
[7] D. Ng, L. Xiang, and R. Schober, "Multi-objective beamforming for secure communication in systems with wireless information and power transfer," in proceedings of the IEEE 24th International Symposium on Personal Indoor and Mobile Radio Communications (PIMRC), 2013.

[8] E. Bedeer, O. Dobre, M. Ahmed, K. Baddour, "A multiobjective optimization approach for optimal link adaptation of OFDM-based cognitive radio systems with imperfect spectrum sensing," IEEE Transactions on Wireless Communications, vol. 13, no. 4, pp. 2339-2351, Apr. 2014.

[9] N. Mokari, H. Saeedi, and K. Navaie, "Channel coding increases the achievable rate of the cognitive networks," IEEE Comm. Letters, vol. 17, pp. 495-498, 2013.
[10] M. G. Khoshkholgh, K. Navaie, and H. Yanikomeroglu, "Access strategies for spectrum sharing in fading environment: Overlay, underlay and mixed," IEEE Trans. Mobile Comput., vol. 9, no. 12, pp. 1780-1793, Dec. 2010.

[11] S. Boyd and L. Vandenberghe, Convex Optimization. Cambridge University Press, 2004.

[12] M. Grant and S. Boyd, CVX Users' Guide for CVX version 1.2 (build 711), 2009, http://www.stanford.edu/ boyd/cvx/download.html.

[13] T. A. Le and M. R. Nakhai, "Coordinated beamforming using semidefinite programming," in Proc. IEEE Int. Conf. Commun., Jun. 2012, pp. $1-5$. 